\title{
P054. Chronic migraine and onabotulinumtoxinA: results from clinical practice
}

\author{
Matteo Paolucci ${ }^{*}$, Riccardo Altavilla', Giulia Gambale ${ }^{1}$, Claudio Altamura', Giovanna Viticchi ${ }^{2}$, Marco Bartolini ${ }^{2}$, \\ Mauro Silvestrini ${ }^{2}$, Fabrizio Vernieri ${ }^{1}$
}

From Abstracts from the 1st Joint ANIRCEF-SISC Congress

Rome, Italy. 29-31 October 2015

\section{Background}

OnabotulinumtoxinA injection according to PREEMPT protocol is a second-line therapy for chronic migraine (CM)[1]. While its efficacy on frequency of headache has been demonstrated[2], our current clinical experience indicates that patients report benefits regardless of reduction of frequency of attacks, as Lipton et al previously showed[3]. The present study aimed at assessing the impact of botulin injection on intensity, quality and perception of pain in patients with $\mathrm{CM}$.

\section{Materials and methods}

We enrolled 25 patients who underwent botulin injections from April 2014 to June 2015. We evaluated patients at baseline (T0, the day of the first botulin injection) and every three months, along with a new injection session. Evaluation consisted in a multiparametric self-assessment questionnaire: pain intensity through 11 point Box Scale (BS-11) and Present Pain Intensity (PPI), changes in functioning through 6-point Behavioral Rating Scale (BRS-6), quality of pain through Short-form McGill Pain Assessment Questionnaire (SF-MPQ) and disability through Migraine Disability Assessment (MIDAS) questionnaire and HIT-6.

At the present time, we have follow-up data for $57 \%$ of patients at three months (T1) and for $38 \%$ of patients at six months (T2).

\section{Results}

We found a global reduction in the intensity of perceived pain and in restriction of activities due to headache: Wilcoxon signed-rank test showed significant reduction in median values of BS-11 at T1 ( $\mathrm{p}$ 0.026) and T3 (p 0.017),

\footnotetext{
* Correspondence: m.paolucci@unicampus.it

${ }^{1}$ Headache Centre, Policlinico Universitario Campus Bio-Medico, Rome, Italy Full list of author information is available at the end of the article
}

of PPI at T1 (p 0.02) and T3 (p 0.046), of BRS-6 at T1 (p 0.008) and T3 (0.026). Analysis of SF-MPQ showed a significant reduction in median values of the descriptors "throbbing" at T1 (p 0.041), "splitting" at T1 (p 0.041) and "tiring-exhausting" at T1 (p 0.011). We did not find significant difference in MIDAS score at T1 and T3 and in frequency of headache in the past three months.

\section{Conclusions}

Our preliminary results show that the OnabotulinumtoxinA usefulness is primarily in improving the quality of life more than in reducing the frequency of headache.

Written informed consent to publication was obtained from the patient(s).

\section{Authors' details}

${ }^{1}$ Headache Centre, Policlinico Universitario Campus Bio-Medico, Rome, Italy. ${ }^{2}$ Clinica Neurologica, Università Politecnica delle Marche, Ancona, Italy.

Published: 28 September 2015

\section{References}

1. Aurora SK, Dodick DW, Turkel CC, DeGryse RE, Silberstein SD, Lipton RB, Diener H-C, Brin MF, PREEMPT 1 Chronic Migraine Study Group: OnabotulinumtoxinA for treatment of chronic migraine: results from the double-blind, randomized, placebo-controlled phase of the PREEMPT 1 trial. Cephalalgia 2010, 30:793-803.

2. Diener H-C, Dodick DW, Aurora SK, Turkel CC, DeGryse RE, Lipton RB, Silberstein SD, Brin MF, PREEMPT 2 Chronic Migraine Study Group: OnabotulinumtoxinA for treatment of chronic migraine: results from the double-blind, randomized, placebo-controlled phase of the PREEMPT 2 trial. Cephalalgia 2010, 30:804-814.

3. Lipton RB, Varon SF, Grosberg B, McAllister PJ, Freitag F, Aurora SK, Dodick DW, Silberstein SD, Diener H-C, DeGryse RE, et al:

OnabotulinumtoxinA improves quality of life and reduces impact of chronic migraine. Neurology 2011, 77:1465-1472.

doi:10.1186/1129-2377-16-S1-A117

Cite this article as: Paolucci et al.: P054. Chronic migraine and onabotulinumtoxinA: results from clinical practice. The Journal of Headache and Pain 2015 16(Suppl 1):A117. 